\title{
Perfil socioeconômico e demográfico e a capacidade funcional de idosos atendidos pela Estratégia Saúde da Família de Paranoá, Distrito Federal
}

\author{
Socioeconomic and demographic profile and functionality of elderly served by the Family Health \\ Strategy in Paranoá, Federal District, Brazil
}

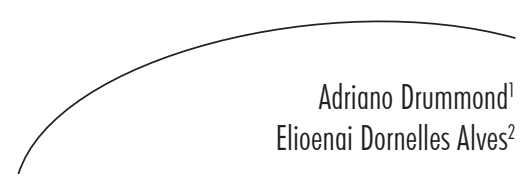

Resumo

Objetivos: Estabelecer o perfil socioeconômico, demográfico e funcional de idosos atendidos pela Estratégia Saúde da Família (ESF) em Paranoá, Distrito Federal, Brasil, e observar as possíveis associações entre os fatores encontrados com o nível de capacidade funcional desta população. Métodos: Trata-se de pesquisa descritiva, com aplicação de questionário socioeconômico e demográfico, escala de Lawton e índice de Katz, para obtenção dos dados sobre funcionalidade em 93 idosos. Para a análise estatística, utilizaram-se o teste exato de Fisher e o qui-quadrado, adotando nível de significância de 0,05. Resultados: Não houve associação entre os elementos de saúde (considerando a autopercepção da saúde, doenças como hipertensão arterial, diabetes, reumatismo, asma, bronquite, varizes, insônia, infarto agudo do miocárdio e acidente vascular encefálico), ou aspectos sociodemográficos e econômicos com funcionalidade, exceto para os fatores histórico de quedas e visita aos amigos $(\mathrm{p}<0,05)$. Conclusão: Houve manutenção principalmente das atividades básicas de vida diária, com pequeno percentual de comprometimento das atividades instrumentais de vida diária.

\section{Abstract}

Objectives: To establish the socioeconomic, demographic and functional profile of the elderly served by the Family Health Strategy (FHS) in Paranoá, Federal District, Brazil, observing possible associations among the factors found with the level of functional capacity in this population. Methods: This is a descriptive research that applied a socioeconomic and demographic questionnaire, Lawton and Katz scales to obtain data on functionality in 93 elderly individuals. For statistical analysis we used the Fisher exact test and the chi-square, adopting a significance level of 0.05. Results:

Palavras-chave: Idoso. Envelhecimento. Saúde da Família. Aptidão Física.
Key words: Elderly. Aging. Family Health. Physical Fitness.

\footnotetext{
Departamento de Fisioterapia, Unidade da Asa Sul. Centro Universitário Euro-Americano. Brasília, DF, Brasil.

Departamento de Enfermagem, Campus Darcy Ribeiro. Universidade de Brasília. Brasília, DF, Brasil.
}

Artigo originário da dissertação de mestrado intitulada Fatores de influência na capacidade funcional de idosos inscritos na Estratégia Saúde da Família no Paranoá-DF, produzido no Grupo de Pesquisa do NESPROM, Universidade de Brasília. 
There was no association between health elements (considering self-perceived health, diseases such as hypertension, diabetes, rheumatism, asthma, bronchitis, varicose veins, insomnia, acute myocardial infarction and stroke), or sociodemographic and economic functionality, except for factors such as history of falls and visiting friends $(\mathrm{p}<0.05)$. Conclusion: There was maintenance of basic activities of daily living, with small percentage of commitment to instrumental activities of daily living.

\section{INTRODUÇÃo}

$\mathrm{O}$ processo de envelhecimento e as doenças crônicas relacionadas à idade podem sobrecarregar o sistema público de saúde e a família, uma vez que compromete a autonomia do indivíduo. $\mathrm{O}$ número de comorbidades associadas à incapacidade funcional é determinante no grau de dependência do idoso. ${ }^{1}$ Algumas doenças, como hipertensão arterial sistêmica (HAS), osteoporose, diabetes e depressão, estão relacionadas ao grau de comprometimento da capacidade funcional; ${ }^{1-4} \mathrm{e}$ aspectos sociais, econômicos e demográficos já foram identificados como associados também à funcionalidade dessa população, como renda, nível educacional e situação conjugal. ${ }^{1,4}$

Os idosos diariamente atendidos por equipes da Estratégia Saúde da Família (ESF) compõem um grupo vulnerável que vem sendo analisado quanto a seu perfil nos aspectos socioeconômicos, demográficos e de saúde, dentre outros objetivos, em algumas regiões do país. ${ }^{2,3,5}$ Esses estudos vêm sendo realizados com o intuito de identificar as características dos usuários que procuram o serviço e ações de saúde promovidos pela ESF, sendo possível orientar, a partir de então, decisões relativas às prioridades de ação. ${ }^{5}$

A ESF evidencia uma forma de se estabelecer uma comunicação diferenciada com a população, uma vez que promove a saúde e prevenção de doenças, e não só cura e reabilitação. Suas contribuições são desenvolvidas de acordo com os problemas e características de cada localidade onde a ESF é implementada. ${ }^{6}$

Diante do exposto, o objetivo desta pesquisa foi estabelecer o perfil socioeconômico, demográfico e funcional de idosos atendidos pela ESF em Paranoá, Distrito Federal, observando possíveis associações entre os fatores encontrados e o nível de capacidade funcional dessa população.

\section{METODOLOGIA}

Trata-se de estudo transversal descritivo, desenvolvido em uma microárea de abrangência da ESF na cidade satélite de Paranoá, Distrito Federal, no período de junho a outubro de 2011. A amostra foi por conveniência, com base no cadastro de 289 inscritos na Unidade Básica de Saúde (UBS) da quadra 18. Foram incluídos indivíduos de ambos os sexos, acima dos 60 anos de idade, que estivessem inscritos na ESF da referida UBS, e excluídos aqueles que não estivessem inscritos, que não fossem encontrados em suas residências ou que não aceitassem participar voluntariamente. Assim sendo, 93 idosos participaram e responderam aos pesquisadores, e 19 foram excluídos.

Os dados socioeconômicos foram pertinentes à escolaridade, renda mensal e situação ocupacional; os demográficos foram: sexo, idade, estado civil, local de residência, composição familiar e situação da propriedade em que vive. Os referentes à saúde: hospitalização nos últimos seis meses; condição de visão e audição; histórico de acidente vascular encefálico, diabetes, hipertensão arterial sistêmica, asma ou bronquite, varizes, insônia e queda. Referentes às relações socais: fazer atividades físicas, ir ao cinema, visitar amigos, visitar parentes, receber visitas, participar de obras religiosas e se gostaria de trabalhar. $\mathrm{E}$, por último, o registro da autopercepção da saúde: estado de saúde (ótima/ 
boa, regular, má/péssima), estado de saúde comparado com a saúde de seus pares (muito pior/pior, a mesma, melhor/muito melhor).

No que se refere à avaliação funcional, a escala de Lawton é um instrumento eficiente para analisar as atividades instrumentais de vida diária (AIVD). ${ }^{5,7,8}$ Composta por oito itens, permite avaliar se uma pessoa pode preparar suas refeições por conta própria, realizar tarefas de casa, lavar as roupas, tomar medicamentos, chegar a locais nos quais é necessário caminhar por longas distâncias, ir à padaria, manusear o dinheiro e usar o telefone.

Para a avaliação das atividades básicas de vida diária (ABVD), foi aplicado o índice de Katz. ${ }^{5,7,9}$ São seis itens analisados e pontuados de acordo com a dependência ou independência: banhar-se, vestir-se, ir ao banheiro, transferir-se, controle sobre sua continência e alimentar-se.

Primeiramente, foram determinadas cinco microáreas de coleta de dados, onde se situavam as residências dos idosos inscritos, cada uma coberta pela ação de um agente comunitário de saúde (ACS), responsável por listar cada idoso participante do programa. Cada idoso foi selecionado aleatoriamente, por sorteio na listagem de cadastro. Caso o idoso não estivesse presente ou não aceitasse participar, a residência mais próxima era eleita como substituta.

A problemática da pesquisa era sempre elucidada antes de qualquer argumentação específica. Em sequência, os aspectos éticos, esclarecendo a confidencialidade dos dados pessoais e o respeito de exposição apenas ao que era relacionado à capacidade funcional. $\mathrm{O}$ questionário e as escalas foram preenchidos pelos pesquisadores após o aceite dos voluntários, nas próprias residências visitadas. Caso o idoso não fosse capaz de responder, um acompanhante o fazia, normalmente um membro da família, morador da mesma residência.

A pesquisa foi aprovada pelo Comitê de Ética em Pesquisa da Fundação de Ensino e Pesquisa em Ciências da Saúde (FEPECS/DF), sob o protocolo $n^{\circ} 149 / 2011$. Os entrevistados foram convidados a participar da pesquisa, sendo esclarecidos sobre os objetivos do projeto por meio da leitura do Termo de Consentimento Livre e Esclarecido (TCLE) e, nos casos de analfabetos, por uma explicação detalhada fornecida pelo pesquisador responsável.

Só participaram aqueles que concordaram em contribuir com as informações solicitadas, assinando o TCLE. Caso o voluntário não soubesse assinar, algum parente ou responsável era indicado para a assinatura do termo. A qualquer momento o voluntário pôde solicitar a exclusão de seus dados fornecidos, o que não ocorreu.

Após a análise descritiva da amostra, coube verificar se alguns desses aspectos socioeconômicos e demográficos se relacionaram com as medidas de capacidade funcional dos indivíduos (escala de Lawton e índice de Katz). Para tal análise, foi utilizado o software SPSS (versão 17.0 para Windows). Os testes utilizados foram o qui-quadrado e o teste exato de Fisher. O nível de significância adotado foi de 0,05.

\section{RESULTADOS}

O perfil socioeconômico e demográfico dos idosos atendidos pela ESF em Paranoá, Distrito Federal, permitiu identificar fatores relativos à saúde e às relações sociais. Com relação aos aspectos socioeconômicos e demográficos, nenhuma associação foi encontrada para a distribuição de medidas de capacidade funcional, já que $95,7 \%$ se encontravam independentes para ABVDs e $75,3 \%$ para AIVDs.

De acordo com os resultados da avaliação socioeconômica e demográfica, os itens que mais se destacaram foram referentes à predominância do sexo feminino, ao número de aposentados e pensionistas, e à faixa de renda dos entrevistados, os quais não percebiam mais que um salário mínimo, considerando o valor de $\mathrm{R} \$ 545,00$, vigente na época da coleta de dados. Mais detalhadamente, visualizam-se os dados socioeconômicos e demográficos na tabela 1. 
Tabela 1 - Distribuição dos idosos segundo dados socioeconômicos e demográficos. Paranoá, DF, 2011.

\begin{tabular}{|c|c|c|}
\hline Variáveis & Categorias & Frequência $(n=93)$ \\
\hline \multicolumn{2}{|l|}{ Sexo } & \\
\hline & Masculino & 38 \\
\hline & Feminino & 55 \\
\hline
\end{tabular}

Faixa etária

60-69

$51-54$

$70-79$

34

80 ou mais

31

12

Estado civil

$\begin{array}{lcc}\text { Casado(a) } & 44 & 47,3 \\ \text { Separado(a) } & 9 & 9,7 \\ \text { Solteiro(a) } & 6 & 6,5 \\ \text { Viúvo(a) } & 34 & 36,6\end{array}$

Escolaridade

$\begin{array}{lcc}\text { Analfabeto(a) } & 28 & 30,1 \\ \text { Até ensino fund.completo } & 60 & 64,5 \\ \text { Até ensino médio completo } & 5 & 5,4\end{array}$

Situação ocupacional

$\begin{array}{lll}\text { Aposentado } & 43 & 46,2 \\ \text { Dona de casa } & 10 & 10,8 \\ \text { Pensionista } & 30 & 32,3 \\ \text { Aposentado/trabalha } & 10 & 10,8\end{array}$

Faixa de renda

Até 1 salário mínimo $\quad 50 \quad 53,8$

De 1 a 2 salários mínimos $\quad 26 \quad 28$

$>2$ salários mínimos $\quad 11 \quad 11,8$

$\begin{array}{lll}\text { Sem renda } & 6 & 6,5\end{array}$

Composição familiar

Apenas cônjuge $13 \quad 13,9$

Cônjuge e filhos $33 \quad 35,4$

Cônjuge e netos 2,1

Cônjuge, filhos e netos $\quad 7 \quad 7,5$

Filhos e netos $24 \quad 25,8$

$\begin{array}{llr}\text { Mora sozinho } & 8 & 8,6\end{array}$

$\begin{array}{lll}\text { Netos } & 4 & 4,3\end{array}$

$\begin{array}{lll}\text { Outros } & 2 & 2,1\end{array}$

Situação de moradia

$\begin{array}{lll}\text { Do entrevistado(a) } & 75 & 80,6\end{array}$

Do cônjuge/filhos $12 \quad 12,9$

Outra situação 44

$\begin{array}{lll}\text { Alugado } & 2 & 2,2\end{array}$ 
Outros aspectos avaliados, relacionados à saúde, podem ser observados na tabela 2. Destacase, nesse contexto, o alto índice de idosos com HAS, com histórico de quedas e/ou que já foram hospitalizados. Em contrapartida, houve baixa prevalência de voluntários que sofreram infarto agudo do miocárdio, asma, bronquite e/ou acidente vascular encefálico. As outras doenças investigadas tiveram um índice praticamente pareado no que diz respeito a quem as apresenta ou não.

Tabela 2 - Condições de saúde dos idosos entrevistados. Paranoá, DF, 2011.

\begin{tabular}{cccc}
\hline Variáveis & Categorias & Frequência $(\mathrm{n}=93)$ & $\%$ \\
\hline
\end{tabular}

Diabetes

$\begin{array}{lll}\text { Sim } & 19 & 20,4 \\ \text { Não } & 74 & 79,6\end{array}$

Acidente vascular encefálico

$\begin{array}{lll}\text { Sim } & 17 & 18,3 \\ \text { Não } & 76 & 81,7\end{array}$

Asma

$\begin{array}{lrr}\text { Sim } & 7 & 7,5 \\ \text { Não } & 86 & 92,5\end{array}$

Bronquite

$\begin{array}{lll}\text { Sim } & 10 & 10,8 \\ \text { Não } & 83 & 89,2\end{array}$

Hipertensão arterial sistêmica

$\begin{array}{lll}\text { Sim } & 67 & 72 \\ \text { Não } & 26 & 28\end{array}$

Insônia

$\begin{array}{lll}\text { Sim } & 38 & 40,9 \\ \text { Não } & 55 & 59,1\end{array}$

Reumatismo

$\begin{array}{lll}\text { Sim } & 39 & 41,9 \\ \text { Não } & 54 & 58,1\end{array}$

Varizes

$\begin{array}{lll}\operatorname{Sim} & 42 & 45,2\end{array}$

$\begin{array}{lll}\text { Não } & 51 & 54,8\end{array}$

Infarto do miocárdio

$\begin{array}{lrc}\text { Sim } & 7 & 7,5 \\ \text { Não } & 86 & 92,5\end{array}$

Histórico de quedas

$\begin{array}{lll}\text { Sim } & 47 & 50,5 \\ \text { Não } & 46 & 49,5\end{array}$

Hospitalizado

$\begin{array}{lll}\text { Sim } & 54 & 58,1 \\ \text { Não } & 39 & 41,9\end{array}$


Quanto à análise da associação entre as medidas de capacidade funcional do indivíduo, detalhadas na tabela 2 , e os fatores relativos à saúde, somente se observou significância entre o índice de Katz e o histórico de quedas, cujo $\mathrm{p}$-valor foi 0,05. Observa-se a seguinte tendência: quem apresentou histórico de quedas possuía maior dependência nas ABVDs.

A autopercepção da saúde, identificada nos dados da tabela 3, é apresentada com alto índice de idosos na condição de "não caso", o que representa aqueles que não possuíam distúrbios cognitivos que interferissem nas respostas. Diante disso, pode-se destacar o item "como percebe sua saúde", em que a maioria relatou estar regular ou boa/ótima. No item "saúde comparada aos seus pares", onde se lê "mais ou menos" como resposta, interpreta-se a condição de que o voluntário percebeu uma similaridade de sua saúde com a de seus pares. Esta resposta obteve um índice pouco inferior à predominante "muito melhor/melhor".

Tabela 3 - Autopercepção da saúde dos idosos entrevistados. Paranoá, DF, 2011.

\begin{tabular}{|c|c|c|c|}
\hline Variáveis & Categorias & Frequência (n=93) & $\%$ \\
\hline \multicolumn{4}{|l|}{ Saúde mental } \\
\hline & Caso & 8 & 8,6 \\
\hline & Não caso & 85 & 91,4 \\
\hline \multicolumn{4}{|l|}{ Como está a visão } \\
\hline & Ótima/boa & 36 & 38,7 \\
\hline & Regular & 32 & 34,4 \\
\hline & Ruim/péssima & 25 & 26,9 \\
\hline \multicolumn{4}{|l|}{ Como está a audição } \\
\hline & Ótima/boa & 58 & 62,4 \\
\hline & Regular & 22 & 23,7 \\
\hline & Ruim/péssima & 13 & 14 \\
\hline \multicolumn{4}{|c|}{ Como percebe sua saúde } \\
\hline & Ótima/boa & 48 & 51,6 \\
\hline & Regular & 34 & 36,6 \\
\hline & Ruim/péssima & 11 & 11,8 \\
\hline \multicolumn{4}{|c|}{ Saúde comparada aos pares } \\
\hline & Muito melhor/melhor & 36 & 38,7 \\
\hline & Mais ou menos & 28 & 30,1 \\
\hline & Muito pior/pior & 25 & 26,9 \\
\hline & Não se aplica & 4 & 4,3 \\
\hline
\end{tabular}

E quanto às relações entre as medidas de capacidade funcional do indivíduo e os fatores relativos às relações sociais, só se observou significância entre o índice de Katz e a visita aos amigos, cujo p-valor foi também 0,05. Ademais, observa-se a seguinte tendência: quem visitava os amigos possuía maior independência nas atividades da vida diária. A tabela 4 apresenta os resultados da avaliação das relações sociais. 
Tabela 4 - Relações sociais dos idosos entrevistados. Paranoá, DF, 2011.

\begin{tabular}{lccc}
\hline \multicolumn{1}{c}{ Relações sociais } & Não & Sim & $\%$ Sim \\
\hline Visita amigos & 74 & 19 & $20,4 \%$ \\
Visita parentes & 76 & 17 & $18,3 \%$ \\
Recebe visitas & 86 & 7 & $7,5 \%$ \\
Vai ao cinema & 83 & 10 & $10,8 \%$ \\
Participa de obras religiosas & 26 & 67 & $72,0 \%$ \\
Gostaria de trabalhar & 55 & 38 & $40,9 \%$ \\
Atividade física & 54 & 39 & $41,9 \%$ \\
\hline
\end{tabular}

E como análise da capacidade funcional, pode-se identificar a tendência dos idosos a serem independentes para o autocuidado, conforme avaliado pelo índice de Katz, e houve maior índice de dependência no que diz respeito à participação de atividades de interação com o meio social, avaliadas pela escala de Lawton (tabela 5).

Tabela 5 - Resultados das medidas de capacidade funcional dos idosos. Paranoá, DF, 2011.

\begin{tabular}{lcclcc}
\hline \multicolumn{2}{c}{ Escala de Lawton } & & \multicolumn{3}{c}{ Índice de Katz } \\
& Frequência & $\%$ & & Frequência & $\%$ \\
AIVD dependente & 12 & 12,9 & ABVD dependente & 4 & 4,3 \\
AIVD independente & 70 & 75,3 & ABVD independente & 89 & 95,7 \\
$\begin{array}{l}\text { AIVD parcialmente } \\
\text { dependente }\end{array}$ & 11 & 11,8 & $\begin{array}{l}\text { ABVD parcialmente } \\
\text { dependente }\end{array}$ & 0 & 0,0 \\
Total & 93 & 100,0 & Total & 93 & 100,0 \\
\hline
\end{tabular}

\section{DISCUSSÃO}

Já se identificou, como atribuição e compromisso das equipes de saúde da família, a necessidade de observar os fatores que podem interferir na autonomia. Tais fatores resgatam a importância de se observar o contexto, tanto quanto aos aspectos físicos, patológicos, mas também da possível associação entre a saúde do ser humano com o meio em que vive. ${ }^{10}$ No cenário da ESF, é esperado encontrar idosos de baixa renda, visto que o programa é implementado em comunidades carentes para que se atenda a uma demanda com restrições de acesso ao serviço, com dificuldade de locomoção, entre outros, além de se tentar estabelecer uma comunicação diferenciada, visando a educação em saúde da população, uma ação preventiva.

Na presente pesquisa, contudo, foi possível detectar que a renda não se associou à autonomia dos voluntários, fator este que pôde ser comprovado na pesquisa de Thumé et al. ${ }^{11} \mathrm{O}$ fator relevante é a diferença amostral dos estudos, uma vez que neste houve a abordagem somente de idosos inscritos em uma unidade básica de saúde, 
e no artigo de Thumé et al. houve uma abordagem nacional. No entanto, a não correlação observada entre esses fatores pode se fundamentar também na condição de que a maior parte da população pesquisada vive em situação de pobreza (mais de $53 \%$ com renda de até um salário mínimo e $28 \%$ com renda de até dois salários mínimos mensais, somando 81,8\% da amostra), indicando uma homogeneidade do grupo. Esse fato corrobora os resultados da pesquisa desenvolvida por Virtuoso Junior \& Guerra, na Região Nordeste do país, a qual não identificou relação entre os fatores destacados, por afirmar situação de pobreza do grupo estudado, caracterizando também uniformidade da renda. ${ }^{12}$

Já em outra esfera analítica, níveis educacionais apresentam correlação direta com o de saúde, como maior incidência de aterosclerose da carótida e de acidente vascular encefálico (AVE). Isso se justifica, segundo Pereira et al., ${ }^{13}$ pelo déficit de informações sobre aspectos preventivos dos hábitos e comportamentos de risco à saúde por parte desta população de baixa renda. De acordo com os resultados ressaltados no presente estudo, mais de $18 \%$ da amostra relataram ter sofrido AVE. Este é um dado que corrobora as evidências ao que se pôde observar sobre o nível educacional dos voluntários, o qual se mostrou baixo.

$\mathrm{Na}$ Cidade do México, contudo, idosos com mais de sete anos de estudo apresentam uma chance de relatar um estado de saúde excelente 4,4 vezes maior que os que possuem menor grau de instrução, fato também evidente na cidade de São Paulo e Santiago, no Chile. Nessas cidades, é confirmado que o nível de instrução influencia positivamente na capacidade de desenvolver uma ABVD. ${ }^{14}$

Nas quadras visitadas em Paranoá, não houve relação do nível de escolaridade dos idosos com a funcionalidade, apesar de a grande maioria $(94,6 \%)$ ser composta de analfabetos ou apresentar nível básico de formação. Muitos relataram ser naturais de outros municípios onde a oportunidade de educação é precária e, portanto, era necessário trabalhar desde a infância ou adolescência para auxiliar no sustento do lar. Assim sendo, foi preciso interromper a formação educacional.

Mas o nível de escolaridade pode influenciar em outras doenças que, consequentemente, podem gerar graus de morbidade e incapacidade funcional, como a HAS e diabetes mellitus. ${ }^{15} \mathrm{Tal}$ fato se confirmou em pesquisa realizada no município de Teixeiras, MG, na qual 150 idosos hipertensos e diabéticos foram entrevistados e foi demonstrado que $89,4 \%$ apresentavam baixo nível educacional, o que levou a uma baixa adesão ao tratamento das doenças, tanto pelas menores condições financeiras (outra característica do grupo estudado) quanto pela falta de conhecimento. Confirmando essa correlação, Banhato \& Guedes concluíram a influência da escolaridade na associação negativa entre cognição e hipertensão com voluntários idosos. ${ }^{15}$ Este é um fato que se confirma com os idosos avaliados em Paranoá, uma vez que a maioria possuía baixa escolaridade e $72 \%$ apresentaram HAS. Apesar de não haver comprometimento funcional deste grupo no momento da avaliação, é preciso que haja atenção quanto a esta doença por parte da equipe de saúde e dos próprios voluntários, para que no futuro haja manutenção da independência.

No intuito de identificar os fatores associados à capacidade funcional (CF) de idosos longevos (80 anos ou mais), autores encontraram funcionalidade ruim entre as mulheres do município de São Geraldo, MG. ${ }^{16}$ Esse resultado corrobora os achados de uma análise baseada em dados nacionais, ${ }^{17}$ indicando, assim, que o sexo feminino é mais vulnerável ao declínio funcional que o masculino. No entanto, em outros estudos onde houve predominância do sexo feminino entre os fatores analisados, esta variável não foi correlacionada com o desempenho funcional, ${ }^{11,18-20}$ assim como na presente pesquisa, onde houve predominância do sexo feminino (65\%), mas não se correlacionou o sexo com o status funcional. Poucos foram os idosos longevos aqui identificados, apenas 12\%, mas nessa estratificação também houve predomínio feminino e a maioria era dependente parcial ou 
totalmente nas AIVDs. Isto revela que, como a ABVD estava praticamente preservada, o comprometimento principal era o da interação do ser com o ambiente externo, social.

A associação entre a funcionalidade e a opinião do idoso de que sua saúde é pior do que a de seus pares, defendida por alguns autores, ${ }^{17}$ não foi fator determinante para outros, ${ }^{16}$ bem como nesta pesquisa.

É comum observar idosos que moram com filhos, netos e/ou acompanhantes, por isso, alguns pesquisadores incluem em suas metodologias esta análise. Nestes termos, há evidências da relação entre a situação conjugal e a CF, sendo melhor entre os $\operatorname{casados}^{11,19}$ ou viúvos, ${ }^{18,20}$ e pior entre separados ou solteiros. Na presente pesquisa, a situação conjugal não foi determinante para a manutenção funcional. Há evidências, entretanto, que indicam a viuvez como característica negativa na condição funcional, diante da influência psicológica da perda familiar, além da redução da condição financeira, como hipóteses que justifiquem tal comprometimento em idosos do município de Jequié, BA. ${ }^{12}$

A participação familiar pode interferir na CF de idosos dependentes quanto à relação de adaptação, companheirismo, desenvolvimento e afetividade da família, uma vez que 73,5\% dos idosos de um estudo relataram comprometimento da dinâmica familiar, sendo que $46 \%$ apresentavam CF moderada e $27 \%$ apresentavam alta disfuncionalidade. ${ }^{18} \mathrm{O}$ conhecimento da dinâmica familiar pode favorecer a compreensão dos mecanismos e recursos de assistência aos idosos, principalmente daqueles mais velhos. Observou-se, durante a coleta de dados, que principalmente os indivíduos acima de 80 anos desta pesquisa necessitavam de maior atenção da família do que os mais novos. E devido à situação econômica, é praticamente impossível contratar um cuidador profissional, sendo a família de fundamental importância para a manutenção e estímulo da CF.

Algumas doenças foram mais prevalentes, destacando-se a HAS, seguida de varizes, reumatismo e insônia. As evidências científicas apontam para uma realidade populacional semelhante, uma vez que a HAS também foi diagnosticada como doença mais prevalente em outras pesquisas, assim como sua interferência na funcionalidade dos idosos. ${ }^{11,17-19,21}$

A artrose e a artrite, por exemplo, podem interferir na mobilidade do idoso, limitando-o quanto ao desempenho de atividades diárias, devido ao próprio processo degenerativo patológico, bem como ao quadro álgico que proporcionam. ${ }^{21} \mathrm{Em}$ estudo realizado com 75 idosos residentes em Goiânia, GO, ${ }^{21}$ 44\% apresentavam limitação para andar, caracterizando interferência na AIVD; 28\% apresentaram déficit no autocuidado para alimentação; 25,35\%, déficit no autocuidado para higiene, e 18,6\%, déficit no autocuidado para vestir-se e arrumar-se, sendo essas tarefas caracterizadas como ABVDs. ${ }^{7}$ Em estudo comparando a Pesquisa Nacional por Amostra de Domicílios (PNAD) de 2003 e 2008, foi possível confirmar a alta prevalência de HAS, seguida de doença de coluna, artrite/reumatismo, depressão e bronquite/asma, todas com índice mais alto em mulheres. Revelou-se ainda que a distribuição de prevalência de uma doença crônica, ao menos, considerando-se idade e sexo, aumenta intensamente até os 70 anos de idade. ${ }^{22}$

$\mathrm{O}$ índice de insônia da presente pesquisa $(40,9 \%)$ se aproxima de outras evidências apontadas em Bambuí, MG $(36,7 \%) .{ }^{23}$ Nesse estudo, mais de 1.600 idosos foram acompanhados por dez anos, e de acordo com o objetivo das autoras, de determinar a incidência e os determinantes de eventos em saúde da população pesquisada, com baixo nível socioeconômico, a conclusão foi que a condição de saúde mais frequente foi HAS e, em quinta colocação, foi a insônia. Tais resultados são similares aos obtidos em Paranoá, em que a HAS foi a mais prevalente e a insônia a quarta mais prevalente, muito embora não tenha havido associação entre tal fator e CF.

No presente estudo, observou-se relação entre histórico de quedas com um maior 
comprometimento funcional. Cabe então salientar que o controle postural é mantido diante da interação dos sistemas sensoriais e neuromotores, permitindo a adaptação diante de uma situação estática, permanecendo parado em pé com ambos os pés apoiados (ortostatismo), ou dinâmica (caminhando ou correndo). ${ }^{24}$ Mas o próprio processo de envelhecimento leva a uma alteração da integridade dos sistemas e da interação entre eles, favorecendo a ocorrência de quedas. As alterações visuais, fraqueza muscular, comprometimento do sistema são fatores intrínsecos de risco de quedas, por exemplo. ${ }^{25,26}$ Os fatores extrínsecos não serão discutidos, uma vez que não foram objetos de investigação nesta pesquisa.

Nesta pesquisa, 61,3\% dos voluntários relataram apresentar visão regular ou ruim/ péssima, corroborando o que a literatura evidencia, lembrando que mais de 50\% dos entrevistados relataram já ter caído. As consequências normalmente são relacionadas aos vários graus de morbidade, e as fraturas são comumente diagnosticadas. A limitação funcional pode ocorrer na atitude de caminhar fora de casa, restringir o uso de transporte público, realizar a higiene pessoal ou até mesmo se agachar, entre outros. ${ }^{27}$

As consequências apontadas permitem analisar que as limitações não só se evidenciam dentro de casa, nas atividades básicas, mas na relação do ser com a sociedade, nas atividades instrumentais. E isto também pôde ser comprovado neste estudo, uma vez que houve relação entre visitar os amigos e o melhor desempenho funcional, conforme já explicitado nos resultados, apesar da baixa porcentagem dos que visitam. Rosa et al., em seu estudo que buscou descrever a distribuição das redes sociais e de apoio em idosos do município de São Paulo, discutem o fato de que a incapacidade funcional impede o fornecimento de ajudas, visto que com o avançar da idade é de se esperar menor desempenho quanto à autonomia. ${ }^{28}$

A prática regular de atividade física é comprovadamente um fator contribuinte para a manutenção da funcionalidade e prevenção de quedas. Isso pode evitar hospitalizações e diferentes graus de morbidade. Idosos com histórico de internações hospitalares apresentam maior vulnerabilidade ao declínio funcional do que os que não foram hospitalizados. ${ }^{21,29}$ Mais de $58 \%$ da amostra apresentaram histórico de hospitalizações e 39\% praticavam atividade física. Foram considerados ativos fisicamente apenas aqueles idosos que fizessem uma prática regular com frequência de pelo menos duas vezes por semana e que completassem 150 minutos de atividades semanais. ${ }^{30}$ Essas são informações que, de acordo com a literatura, seriam suficientes para determinar uma população mais vulnerável ou dependente funcionalmente, diante de uma maioria não ativa. Isso não pôde ser observado, uma vez que o que se encontrou foi um alto índice de manutenção funcional, total ou parcialmente, tanto para ABVDs quanto para AIVDs. Contudo, o que se observou durante as entrevistas foi que os voluntários realizavam atividades dentro de casa, como alimentação, manutenção e limpeza do lar, por exemplo, caracterizando estímulo à mobilidade. A mobilidade corporal é fundamental para que se mantenham as ABVDs.

Com base no que foi exposto, ressalta-se que a amostra deste estudo é um fator limitante, pois não caracteriza uma representatividade de dados populacionais brasileiros, bem como possivelmente contribuiu para divergir de fatores associados à capacidade funcional encontrados em outras pesquisas nacionais.

\section{CONSIDERAÇÕES FINAIS}

O perfil dos voluntários se destaca pelo predomínio do sexo feminino, casados, que moram com cônjuges e filhos, de moradia própria, com renda de até um salário mínimo, aposentados, com formação educacional até o ensino fundamental completo, e na faixa etária dos 60-69 anos. É importante salientar, ainda, que a maior parte dos entrevistados apresenta HAS, o que pode representar futura complicação funcional, pois se sabe que é uma 
doença que pode levar a sérias consequências físicas e fisiológicas e até mesmo a outras doenças, gerando limitação funcional.

Destacam-se, assim, os dois únicos fatores de influência na funcionalidade dos idosos na microárea de Paranoá: 1) histórico de quedas; e 2) visitar os amigos, pois aqueles que relataram fazer isso apresentaram maior sociabilidade, indicando melhor relação com as AIVDs e maior independência.

O comprometimento maior, sobretudo dos idosos parcialmente dependentes, foi relativo às AIVDs, com maior preservação das ABVDs, indicando maior tempo de atividades em suas residências, com menor dedicação à participação social.

\section{REFERÊNCIAS}

1. Rosa TEC, Benício MHD, Latorre MRDO, Ramos LR. Fatores determinantes da capacidade funcional entre idosos. Rev Saúde Pública 2003;37(1):40-8.

2. Linhares CRC, Coelho VLD, Guimarães RM, Campos APM, Carvalho NT. Perfil da clientela de um ambulatório de geriatria do Distrito Federal. Psicol Reflex Crit 2003;16(2):319-26.

3. Camarano AA. Mecanismos de proteção social para a população idosa brasileira. Textos para discussão n. 1179. Rio de Janeiro: Instituto de Pesquisa Econômica Aplicada; 2006.

4. Cotta RM, Batista KC, Reis RS, de Souza GA, Dias G, de Castro FA, et al. Social-sanitary and lifestyle profile of hypertense and/or diabetics, users of the Family Health Program in the city of Teixeiras, Minas Gerais State. Cienc Saude Colet 2009;14(4):1251-60.

5. Garcia ESS, Saintrain MVL. Perfil epidemiológico de uma população idosa atendida pelo Programa Saúde da Família. Rev enferm UERJ 2009;17(1):18-23.

6. Araújo LAO, Bachion MM. Programa Saúde da Família: perfil de idosos assistidos por uma equipe. Rev Bras Enferm 2004;57(5):586-90.

7. Shiguemoto GE. Avaliação do idoso: anamnese e exame da performance física. In: Rebelatto JR, Morelli JGS, organizadores. Fisioterapia geriátrica - a prática de assistência ao idoso. Barueri: Manole; 2007. p. 95-97.

8. Lawton MP, Brody EM. Assessment of older people: self-maintaining and instrumental activities of daily living. Gerontologist 1969;9(3):179-86.

9. Duarte YAO, Andrade CL, Lebrão ML. O índex de Katz na avaliação da funcionalidade dos idosos. Rev Esc Enferm USP 2007;41(2):317-25.

10. Silvestre JA, da Costa Neto MM. Approach to the elderly in family health programs. Cad Saúde Pública 2003;19(3):839-47.
11. Thumé E, Facchini LA, Tomasi E, Vieira LA. Home health care for the elderly: associated factors and characteristics of access and health care. Rev Saúde Pública 2010;44(6):1102-11.

12. Virtuoso Junior JS, Guerra RO. Fatores associados às limitações funcionais em idosos de baixa renda. Rev Assoc Med Bras 2008;54(5):430-5.

13. Pereira ABCNG, Alvarenga $H$, Pereira Junior RS, Barbosa MTS. Prevalência de acidente vascular cerebral em idosos no Município de Vassouras, Rio de Janeiro, Brasil, através do rastreamento de dados do Programa Saúde da Família. Cad Saúde Pública 2009;25(9):1929-36.

14. Noronha KVMS, Andrade MV. Desigualdades sociais em saúde e na utilização dos serviços de saúde entre os idosos na América Latina. Rev Panam Salud Publica 2005;17(5-6):410-8.

15. Banhato EFC, Guedes DV. Cognição e hipertensão: influência da escolaridade. Estud Psicol 2011;28(2):143-51.

16. Nogueira SL, Ribeiro RCL, Rosado LEFPL, Franceschini SCC, Ribeiro AQ, Pereira ET. Determinant factors of functional status among the oldest old. Rev Bras Fisioter 2010;14(4):322-9.

17. Alves LC, Leite IC, Machado CJ. Factors associated with functional desability of elderly in Brazil: a multilevel analysis. Rev Saúde Pública 2010;44(3):468-78.

18. Torres GV, Reis LA, Reis LA, Fernandes MH, Xavier T'T. Relação entre funcionalidade familiar e capacidade funcional de idosos dependentes no município de Jequié (BA). Rev Baiana Saúde Pública 2010;34(1):19-30.

19. Costa EC, Nakatani AYK, Bachion MM. Elder's community capacity to develop daily life activities and daily instrumental life activities. Acta Paul Enferm 2006;19(1):35-43. 
20. Ursine PG, Cordeiro Hde A, Moraes CL. Prevalence of housebound elderly people in the urban region of Belo Horizonte (Minas Gerais, Brasil). Cien Saude Colet 2011;16(6):2953-62.

21. Araújo LA, Bachion MM. Nursing diagnoses of the pattern of mobility in the elderly attended by the Family Health Program. Rev Esc Enferm USP 2005;39(1):53-61.

22. Barros MBA, Francisco PMSB, Zanchetta LM, César CLG Tendências das desigualdades sociais e demográficas na prevalência de doenças crônicas no Brasil, PNAD: 2003-2008. Cien Saude Colet 2011;16(9):3755-68.

23. Lima-Costa MF, Firmo JO, Uchôa E. The Bambuí Cohort Study of Aging: methodology and health profile of participants at baseline. Cad Saúde Pública 2011;27(Suppl 3):S327-35

24. Schumway-Cook A, Woollacott MH. Controle postural. In: Schumway-Cook A, Woollacott MH, organizadores. Controle Motor: teoria e aplicações práticas. Barueri: Manole; 2003. p.153-178.

25. Pinho L, Dias RC, Freire MTF, Tavares CF, Dias JMD . Avaliação isocinética da função muscular do quadril e tornozelo em idosos que sofrem quedas. Rev Bras Fisioter 2005;9(1):93-9.

26. Ribeiro ASB, Pereira JS. Melhora do equilíbrio e redução da possibilidade de queda em idosas após os exercícios de Cawthorne e Cooksey. Rev Bras otorrinolaringol 2005;71(1):38-46.

27. Fabrício SCC, Rodrigues RAP, Costa Junior ML. Causas e conseqüências de quedas em idosos atendidos em hospital público. Rev Saúde Pública 2004;38(1):93-9.

28. Rosa TEC, Benício MHD, Alves MCGP, Lebrão ML. Aspectos estruturais e funcionais do apoio social de idosos do Município de São Paulo, Brasil. Cad Saúde Pública 2007;23(12):2982-92.

29. Silva TO, Freitas RS, Monteiro MR, Borges SM. Avaliação da capacidade física e quedas em idosos ativos e sedentários da comunidade. Rev Bras Clin Med 2010;8(5):392-8.

30. Craig CL, Marshall AL, Sjöström M, Bauman AE, Booth ML, Ainsworth BE, et al. International physical activity questionnaire: 12-country reliability and validity. Med Sci Sports Exerc 2003;35(8):1381-95. 